Kragujevac Journal of Mathematics

Volume 39(1) (2015), Pages 73-82.

\title{
ON A NEW MODEL OF NONLOCAL MODIFIED GRAVITY
}

\author{
JELENA GRUJIC ${ }^{1}$
}

\begin{abstract}
Nonlocal modified gravity without matter, where nonlocality is of the form $R^{-p} \mathcal{F}(\square) R$ is considered from the cosmological point of view. Equations of motion are derived. Cosmological solutions of the form $a(t)=a_{0}\left|t-t_{0}\right|^{\alpha}$, for the FLRW metric and curvature constant $k=0, \pm 1$, are found.
\end{abstract}

\section{INTRODUCTION}

General Relativity is the Einstein theory of gravity usually given in the form of the equation of motion for gravitational (metric) field $g_{\mu \nu}$, i.e. $R_{\mu \nu}-\frac{1}{2} R g_{\mu \nu}=8 \pi G T_{\mu \nu}$, where $R_{\mu \nu}$ is the Ricci curvature tensor, $R$ is scalar curvature, $T_{\mu \nu}$ is the energymomentum tensor, and speed of light is taken $c=1$. This Einstein equation can be derived from the Einstein-Hilbert action $S=\frac{1}{16 \pi G} \int \sqrt{-g} R d^{4} x+\int \sqrt{-g} L_{m} d^{4} x$, where $g=\operatorname{det}\left(g_{\mu \nu}\right)$ and $L_{m}$ is Lagrangian of matter.

Despite its theoretical beauty and many phenomenological evidences, general relativity is not a complete theory and should be modified. There are many its modifications, which are motivated by quantum gravity, string theory, astrophysics and cosmology (for a review, see [1]). One of very promising directions of research is nonlocal modified gravity and its applications to cosmology (as a review, see [2], [3] and [4]). It usually contains an infinite number of spacetime derivatives in the form of some power expansions of the d'Alembert operator $\square=\frac{1}{\sqrt{-g}} \partial_{\mu} \sqrt{-g} g^{\mu \nu} \partial_{\nu}$ or of its inverse $\square^{-1}$, or a combination of both. We are mainly interested in nonlocality expressed in the form of an analytic function $\mathcal{F}(\square)=\sum_{n=0}^{\infty} f_{n} \square^{n}$. For nonlocal gravity with $\square^{-1}$ see also $[17,3,18,19]$. Nonlocality also improves renormalizability of gravity, see [20, 21]

Key words and phrases. Modified gravity, nonlocal gravity, power-law cosmological solutions. 2010 Mathematics Subject Classification. Primary: 83D05. Secondary: 83F05, 53B50.

Received: March 3, 2015

Accepted: March 19, 2015. 
and references therein. Note that there have been some investigations with $R^{-1}$ modification of gravity, but they are not nonlocal and they have problems to be confirmed for the Solar System [16].

To solve cosmological Big Bang singularity, nonlocal gravity with replacement $R \rightarrow R+R \mathcal{F}(\square) R$ in the Einstein-Hilbert action was proposed in [5]. This nonlocal model is further elaborated in the series of papers $[6,7,8,9,10,11,12]$.

We are interesting here in nonlocal gravity action without matter in the form

$$
S=\int\left(\frac{R-2 \Lambda}{16 \pi G}+R^{-p} \mathcal{F}(\square) R\right) \sqrt{-g} d^{4} x
$$

where $R$ is scalar curvature, $p \in \mathbb{N}, \mathcal{F}(\square)=\sum_{n=0}^{\infty} f_{n} \square^{n}$ is an analytic function of the d'Alembert-Beltrami operator $\square=\frac{1}{\sqrt{-g}} \partial_{\mu} \sqrt{-g} g^{\mu \nu} \partial_{\nu}, g=\operatorname{det}\left(g_{\mu \nu}\right)$.

The action (1.1) for $p=1$ was introduced in [13] as a new approach to nonlocal gravity. Our intention is to present some power-law cosmological solutions as a part of a systematic investigation of nonlocal gravity (1.1). For $p=1$, in $[13,14]$ similar power-law cosmological solutions were obtained.

\section{Equations of Motion}

By variation of action (1.1) with respect to metric $g^{\mu \nu}$ one obtains the equations of motion for $g_{\mu \nu}$

$$
\begin{aligned}
& \frac{1}{16 \pi G}\left(G_{\mu \nu}+\Lambda g_{\mu \nu}\right)-\frac{1}{2} g_{\mu \nu} R^{-p} \mathcal{F}(\square) R+\left(R_{\mu \nu} \Phi-K_{\mu \nu} \Phi\right) \\
& \quad+\frac{1}{2} \sum_{n=1}^{\infty} f_{n} \sum_{l=0}^{n-1}\left(g_{\mu \nu} \partial^{\alpha} \square^{l} R^{-p} \partial_{\alpha} \square^{n-1-l} R+g_{\mu \nu} \square^{l} R^{-p} \square^{n-l} R\right. \\
& \left.\quad-2 \partial_{\mu} \square^{l} R^{-p} \partial_{\nu} \square^{n-1-l} R\right)=0,
\end{aligned}
$$

where $K_{\mu \nu}=\nabla_{\mu} \nabla_{\nu}-g_{\mu \nu} \square, \quad \Phi=-p R^{-p-1} \mathcal{F}(\square) R+\mathcal{F}(\square) R^{-p}$.

In the case of the FLRW metric, equation (2.1) is equivalent to its trace and 00 component, respectively:

$$
\begin{aligned}
& \frac{1}{16 \pi G}(4 \Lambda-R)-2 R^{-p} \mathcal{F}(\square) R+(R \Phi+3 \square \Phi) \\
& \quad+\sum_{n=1}^{\infty} f_{n} \sum_{l=0}^{n-1}\left(\partial^{\mu} \square^{l} R^{-p} \partial_{\mu} \square^{n-1-l} R+2 \square^{l} R^{-p} \square^{n-l} R\right)=0,
\end{aligned}
$$




$$
\begin{aligned}
& \frac{1}{16 \pi G}\left(G_{00}+\Lambda g_{00}\right)-\frac{1}{2} g_{00} R^{-p} \mathcal{F}(\square) R+\left(R_{00} \Phi-K_{00} \Phi\right) \\
& \quad+\frac{1}{2} \sum_{n=1}^{\infty} f_{n} \sum_{l=0}^{n-1}\left(g_{00} \partial^{\alpha} \square^{l} R^{-p} \partial_{\alpha} \square^{n-1-l} R+g_{00} \square^{l} R^{-p} \square^{n-l} R\right. \\
& \left.\quad-2 \partial_{0} \square^{l} R^{-p} \partial_{0} \square^{n-1-l} R\right)=0 .
\end{aligned}
$$

Equations (2.2) and (2.3) are more suitable for further investigation than (2.1).

In the FLRW metric $d s^{2}=-d t^{2}+a^{2}(t)\left(\frac{d r^{2}}{1-k r^{2}}+r^{2} d \theta^{2}+r^{2} \sin ^{2} \theta d \phi^{2}\right)$ one has $R=6\left(\frac{\ddot{a}}{a}+\frac{\dot{a}^{2}}{a^{2}}+\frac{k}{a^{2}}\right)$ and $\square h(t)=-\ddot{h}(t)-3 H \dot{h}(t)$, where $H=\frac{\dot{a}}{a}$ is the Hubble parameter. In the sequel we solve equations of motions (2.2) and (2.3) for cosmological scale factor $a(t)$ and the corresponding $R$ :

$$
\begin{aligned}
a(t) & =a_{0}\left|t-t_{0}\right|^{\alpha}, \\
R(t) & =6\left(\alpha(2 \alpha-1)\left(t-t_{0}\right)^{-2}+\frac{k}{a_{0}^{2}}\left(t-t_{0}\right)^{-2 \alpha}\right) .
\end{aligned}
$$

\section{CASE $k=0, \alpha \neq 0$ AND $\alpha \neq \frac{1}{2}$}

In this case, there is the following dependence on parameter $\alpha$ :

$$
\begin{aligned}
a & =a_{0}\left|t-t_{0}\right|^{\alpha}, & H & =\frac{\alpha}{t-t_{0}}, \\
R & =Q\left(t-t_{0}\right)^{-2}, & Q & =6 \alpha(2 \alpha-1), \\
R_{00} & =3 \alpha(1-\alpha)\left(t-t_{0}\right)^{-2}, & G_{00} & =3 \alpha^{2}\left(t-t_{0}\right)^{-2} .
\end{aligned}
$$

Now expressions $\square^{n} R^{-p}$ and $\square^{n} R$ become

$$
\begin{aligned}
\square^{n} R^{-p} & =B(n, p)\left(t-t_{0}\right)^{2 p-2 n}, \quad \square^{n} R=B(n,-1)\left(t-t_{0}\right)^{-2-2 n}, \\
B(n, p) & =Q^{-p} 2^{n} \prod_{l=1}^{n}(p-l+1)(-2 p-3 \alpha+2 l-1), n \geq 1, \\
B(n,-1) & =Q 2^{n} \prod_{l=1}^{n}(-l)(1-3 \alpha+2 l), n \geq 1, \\
B(0, p) & =Q^{-p}, \quad B(0,-1)=Q .
\end{aligned}
$$

Also, we have

$$
\mathcal{F}(\square)\left(R^{-p}\right)=\sum_{n=0}^{\infty} f_{n} B(n, p)\left(t-t_{0}\right)^{2 p-2 n} .
$$


Coefficients $B(n, s)$ we can transform in the following way:

$$
\begin{aligned}
B(n, s) & =Q^{-s}(-4)^{n} \frac{\Gamma(s+1) \Gamma(s+\eta+1)}{\Gamma(s+1-n) \Gamma(s+\eta+1-n)}, \quad s \neq-1, \\
B(p,-1) & =Q 4^{p} p ! \frac{\Gamma(\eta)}{\Gamma(\eta-p)}
\end{aligned}
$$

where $\eta=\frac{3 \alpha-1}{2}$. Substituting these equations into (2.2) and (2.3) one has

$$
\begin{aligned}
& \sum_{n=0}^{\infty} f_{n} B(n, p)(Q-6(p-n)(2 p-2 n-1)-18 \alpha(p-n)) \tau \\
& \quad+Q^{-p-1} \sum_{n=0}^{\infty} f_{n} B(n,-1)((-2-p) Q+6 p(p-n)(2 p-2 n-1+3 \alpha)) \tau \\
& \quad+2 \sum_{n=1}^{\infty} f_{n} A_{n} \tau=\frac{1}{16 \pi G}\left(Q\left(t-t_{0}\right)^{-2}-4 \Lambda\right)
\end{aligned}
$$

and

$$
\begin{aligned}
& 3 \alpha \sum_{n=0}^{\infty} f_{n} B(n, p)(1-\alpha+2(p-n)) \tau \\
& \quad+Q^{-p} \sum_{n=0}^{\infty} f_{n} B(n,-1)\left(\frac{1}{2}-3 \alpha(1-\alpha) p Q^{-1}-6 \alpha p Q^{-1}(p-n)\right) \tau \\
& \quad-\frac{1}{2} \sum_{n=1}^{\infty} f_{n} B_{n} \tau=\frac{1}{16 \pi G}\left(\Lambda-3 \alpha^{2}\left(t-t_{0}\right)^{-2}\right)
\end{aligned}
$$

where

$$
\begin{aligned}
A_{n} & =\sum_{l=0}^{n-1} B(l, p)(B(n-l,-1)-2(p-l)(l-n) B(n-l-1,-1)) \\
B_{n} & =\sum_{l=0}^{n-1} B(l, p)(B(n-l,-1)+4(p-l)(l-n) B(n-l-1,-1)), \\
\tau & =\left(t-t_{0}\right)^{2 p-2 n-2} .
\end{aligned}
$$

From equations (3.3) and (3.4) it follows that if $i \neq p, p-1$ we have $f_{i}=0$. Coefficients $f_{p}$ and $f_{p-1}$ have to satisfy the following systems: 


$$
\begin{aligned}
f_{p}\left(K_{1}+S_{1}\right) & =\frac{Q}{16 \pi G}, \\
f_{p}\left(K_{2}+\frac{1}{2} S_{2}\right) & =\frac{-3 \alpha^{2}}{16 \pi G}, \\
f_{p-1}\left(K_{3}+S_{3}\right) & =\frac{-\Lambda}{4 \pi G}, \\
f_{p-1}\left(K_{4}+\frac{1}{2} S_{4}\right) & =\frac{\Lambda}{16 \pi G},
\end{aligned}
$$

where

$$
\begin{aligned}
K_{1} & =(-p-2) Q^{-p} B(p,-1)+Q B(p, p), \\
S_{1} & =\sum_{l=0}^{p-1} 2 B(l, p)\left(2(l-p)^{2} B(-l+p-1,-1)+B(p-l,-1)\right), \\
K_{2} & =\left(3(\alpha-1) \alpha p Q^{-1}+\frac{1}{2}\right) Q^{-p} B(p,-1)+3(1-\alpha) \alpha B(p, p), \\
S_{2} & =\sum_{l=0}^{p-1} B(l, p)\left(4(p-l)^{2} B(-l+p-1,-1)-B(p-l,-1)\right), \\
K_{3}= & \left(6(3 \alpha+1) p Q^{-1}-p-2\right) Q^{-p} B(p-1,-1)+Q B(p-1, p)+3 B(p, p), \\
S_{3}= & \sum_{l=0}^{p-2} 2 B(l, p)(2(l-p)(l-p+1) B(-l+p-2,-1)+B(-l+p-1,-1)), p \neq 1, \\
K_{4}= & \left(3(\alpha-1) \alpha p-6 \alpha p+\frac{Q}{2}\right) Q^{-p-1} B(p-1,-1)+3(3-\alpha) \alpha B(p-1, p), \\
S_{4}= & \sum_{l=0}^{p-2} B(l, p)(4(l-p)(l-p+1) B(-l+p-2,-1)-B(-l+p-1,-1)), p \neq 1 .
\end{aligned}
$$

For $p=1$ we have $S_{3}=S_{4}=0$.

The systems (3.7) and (3.8) have a solution if and only if

$$
\begin{aligned}
-3 \alpha^{2} K_{1}-Q K_{2} & =3 \alpha^{2} S_{1}+\frac{1}{2} Q S_{2}, \\
K_{3}+4 K_{4} & =-\left(S_{3}+2 S_{4}\right) .
\end{aligned}
$$

Using (3.2) we can show that last conditions are satisfied for all $\alpha \in \mathbb{R} \backslash\left\{0, \frac{1}{2}\right\}$ and $p \in \mathbb{N}$. 


\section{CASE $k=0, \alpha \rightarrow 0 \quad$ (Minkowski SpaCe)}

Substituting (3.1) and (3.5) into trace equation (3.3) we obtain

$$
\begin{aligned}
& (6 \alpha(2 \alpha-1))^{-p}\left(\sum_{n=0}^{\infty} f_{n} \widetilde{B}(n, p)(6 \alpha(2 \alpha-1)-6(p-n)(2 p-2 n-1)-18 \alpha(p-n)) \tau\right. \\
& +\sum_{n=0}^{\infty} f_{n} \widetilde{B}(n,-1)((-2-p) 6 \alpha(2 \alpha-1)+6 p(p-n)(2 p-2 n-1)+18 \alpha p(p-n)) \tau \\
& \left.+12 \alpha(2 \alpha-1) \sum_{n=1}^{\infty} f_{n} \sum_{l=0}^{n-1} \widetilde{B}(l, p)(\widetilde{B}(n-l,-1)-2(p-l)(l-n) \widetilde{B}(n-l-1,-1)) \tau\right) \\
& (4.1) \\
& =\frac{1}{16 \pi G}\left(6 \alpha(2 \alpha-1)\left(t-t_{0}\right)^{-2}-4 \Lambda\right),
\end{aligned}
$$

where

$$
\begin{aligned}
\widetilde{B}(n, p) & =2^{n} \prod_{l=1}^{n}(p-l+1)(-2 p-3 \alpha+2 l-1), n \geq 1, \quad \widetilde{B}(0, p)=1, \\
\widetilde{B}(n,-1) & =2^{n} \prod_{l=1}^{n}(-l)(1-3 \alpha+2 l), n \geq 1, \quad \widetilde{B}(0,-1)=1 \\
\tau & =\left(t-t_{0}\right)^{2 p-2 n-2}
\end{aligned}
$$

Now, if $\alpha \rightarrow 0$ from (4.1) we get

$$
\sum_{n=1}^{\infty} f_{n} D_{n} 2^{n+1}(p-n)(2 p-2 n-1)\left(t-t_{0}\right)^{2 p-2 n-2}=0
$$

where

$$
D_{n}=p \prod_{l=1}^{n}(-l)(1+2 l)-\prod_{l=1}^{n}(p-l+1)(-2 p+2 l-1) .
$$

From this we conclude

$$
f_{p-1}, f_{p} \in \mathbb{R}, \quad f_{i}=0, i \neq p-1, p .
$$

Substituting $f_{i}=0, i \neq p-1, p$ into equation (3.4) we obtain the following equations: 


$$
\begin{aligned}
& ((-1-p) \widetilde{B}(p,-1)+\widetilde{B}(p, p) \\
& \left.\quad+\sum_{l=0}^{p-1} \widetilde{B}(l, p)\left(\widetilde{B}(p-l,-1)-4(p-l)^{2} \widetilde{B}(p-l-1,-1)\right)\right) f_{p}\left(t-t_{0}\right)^{-2}=0, \\
& ((-1-3 p) \widetilde{B}(p-1,-1)+3 \widetilde{B}(p-1, p) \\
& \left.\quad+\sum_{l=0}^{p-2} \widetilde{B}(l, p)(\widetilde{B}(p-l-1,-1)+4(p-l)(l-p+1) \widetilde{B}(p-l-2,-1))\right) f_{p-1}=0 .
\end{aligned}
$$

When $\alpha \rightarrow 0$ the corresponding solution is

$$
f_{p} \in \mathbb{R}, \quad f_{i}=0, i \neq p .
$$

Since all $f_{n}=0, n \neq p$ this is not nonlocal gravity model (1.1). It follows that the above power-law cosmological solutions have not Minkowski space as their background, or in other words, they cannot be obtained as perturbations on Minkowski space.

Remark. For $p \neq 1$, case $k=0, \alpha \rightarrow \frac{1}{2}$ does not yield solution which satisfies the equations of motion. For $p=1$ we have solution (see [14]).

\section{CASE $k \neq 0$}

In order to simplify expression (2.5) there are three possibilities: $\alpha=0, \alpha=\frac{1}{2}$ and $\alpha=1$. For $\alpha=\frac{1}{2}$ we have no solutions which satisfy the equations of motion.

5.1. Case $\alpha=0$. In the case $\alpha=0$ we obtain

$$
\begin{gathered}
a=a_{0}, \quad R=\frac{6 k}{a_{0}^{2}}, \\
H=0, \quad \square R=0 .
\end{gathered}
$$

We have $\Phi=(1-p) R^{-p} f_{0}$.

Substituting (5.1) into equations of motion we get the following system of equations:

$$
\begin{aligned}
(-p-1) R^{1-p} f_{0} & =\frac{R}{16 \pi G}-\frac{\Lambda}{4 \pi G}, \\
R^{1-p} f_{0} & =\frac{\Lambda}{8 \pi G}-\frac{R}{16 \pi G}, \\
f_{i} & \in \mathbb{R}, i \neq 0 .
\end{aligned}
$$

The previous system has solution if and only if

$$
32 \pi G \Lambda(1-p)=-p R .
$$

Obviously, for $p=1$ the last condition is not satisfied. 
5.2. Case $\alpha=1$. In the case $\alpha=1$ we obtain

$$
\begin{aligned}
a & =a_{0}\left|t-t_{0}\right|, \quad R=6\left(1+\frac{k}{a_{0}^{2}}\right)\left(t-t_{0}\right)^{-2}, \\
H & =\frac{1}{t-t_{0}}, \quad \square R=0, \\
R_{00} & =0, \quad G_{00}=3\left(1+\frac{k}{a_{0}^{2}}\right)\left(t-t_{0}\right)^{-2},
\end{aligned}
$$

and

$$
\begin{aligned}
& \square^{n} R^{-p}=E(n, p)\left(t-t_{0}\right)^{2 p-2 n}, \\
& E(n, p)=\left(6+\frac{6 k}{a_{0}^{2}}\right)^{-p} 2^{n} \prod_{l=1}^{n}(p-l+1)(-2 p+2 l-4), n \geq 1, \\
& E(0, p)=\left(6+\frac{6 k}{a_{0}^{2}}\right)^{-p}, \\
& \square^{n} R=E(n,-1)\left(t-t_{0}\right)^{-2-2 n}=0, \quad n \geq 1 .
\end{aligned}
$$

Also, we have

$$
\mathcal{F}(\square)\left(R^{-p}\right)=\sum_{n=0}^{\infty} f_{n} E(n, p)\left(t-t_{0}\right)^{2 p-2 n} .
$$

Using the above expressions, from equations of motion it follows that coefficients $f_{p}$ and $f_{p-1}$ have to satisfy the following systems of equations:

$$
\begin{aligned}
(4 E(p-1, p)+E(p, p)) f_{p} & =\frac{1}{16 \pi G}, \\
4 E(p-1, p) f_{p} & =-\frac{1}{16 \pi G}
\end{aligned}
$$

and

$$
\begin{aligned}
\left(48\left(1+\frac{k}{a_{0}^{2}}\right) E(p-2, p)+6\left(1+\frac{k}{a_{0}^{2}}\right) E(p-1, p)+3 E(p, p)\right) f_{p-1} & =-\frac{\Lambda}{4 \pi G} \\
6\left(4\left(1+\frac{k}{a_{0}^{2}}\right) E(p-2, p)+E(p-1, p)\right) f_{p-1} & =\frac{\Lambda}{16 \pi G}, \quad p \neq 1 .
\end{aligned}
$$

We obtain

$$
\begin{aligned}
& f_{n}=0, \quad 0 \leq n \leq p-2 \quad(p \neq 1), \\
& f_{n} \in \mathbb{R}, \quad n \geq p+1 .
\end{aligned}
$$

The systems (5.2) and (5.3) have solution if and only if

$$
\begin{aligned}
& 8 E(p-1, p)=-E(p, p), \\
& 48\left(1+\frac{k}{a_{0}^{2}}\right) E(p-2, p)+\left(10+\frac{2 k}{a_{0}^{2}}\right) E(p-1, p)+E(p, p)=0 .
\end{aligned}
$$


After short calculation we obtain that the last two conditions are satisfied for all $p \neq 1$.

For $p=1$ system (5.3) (which corresponds to coefficient $f_{0}$ ) becomes:

$$
f_{0}=\frac{\Lambda}{8 \pi G} .
$$

Therefore, equations of motion are satisfied for all $p \in \mathbb{N}$.

\section{Concluding Remarks}

In this paper we have presented some power-law cosmological solutions of the form $a(t)=a_{0}\left|t-t_{0}\right|^{\alpha}$, which are derived from modified gravity with nonlocal term $R^{-p} \mathcal{F}(\square) R$. These solutions do not have Minkowski space background. However, for $p=1$ there is the de Sitter bounce solution $a(t)=a_{0} \exp (\lambda t)$, which in the limit $\lambda \rightarrow 0$ leads to the Minkowski space (see $[14,15]$ ).

It is worth noting that there is solution $a(t)=\left|t-t_{0}\right|$ which corresponds to the Milne universe for $k=-1$.

Note also that all the above presented power-law solutions $a(t)=a_{0}\left|t-t_{0}\right|^{\alpha}$ have scalar curvature $R(t)=6\left(\alpha(2 \alpha-1)\left(t-t_{0}\right)^{-2}+\frac{k}{a_{0}^{2}}\left(t-t_{0}\right)^{-2 \alpha}\right)(2.5)$, which satisfies relation $\square R=q R^{2}$, where parameter $q$ depends on $\alpha$. This quadratic relation $\square R=q R^{2}$ was used in [13] as an Ansatz to solve equations of motion for $p=1$.

Acknowledgment: Work on this paper was supported by Ministry of Education, Science and Technological Development of the Republic of Serbia, grant No 174012. I would like to thank Branko Dragovich, Zoran Rakic and Ivan Dimitrijevic for useful discussions.

\section{REFERENCES}

[1] T. Clifton, P. G. Ferreira, A. Padilla and C. Skordis, Modified gravity and cosmology, Phys. Rep. 513 (2012), 1-189.

[2] S. Nojiri and S. D. Odintsov, Unified cosmic history in modified gravity: from $F(R)$ theory to Lorentz non-invariant models, Phys. Rep. 505 (2011), 59-144.

[3] R. P. Woodard, Nonlocal models of cosmic acceleration, Found. Phys. 44 (2014), 213-233.

[4] B. Dragovich, On nonlocal modified gravity and cosmology, in: Lie Theory and Its Applications in Physics, Springer Proceedings in Mathematics and Statistics, 2014, pp. 251-262.

[5] T. Biswas, A. Mazumdar and W. Siegel, Bouncing universes in string-inspired gravity, J. Cosmology Astropart. Phys. 0603 (2006), 009.

[6] T. Biswas, T. Koivisto and A. Mazumdar, Towards a resolution of the cosmological singularity in non-local higher derivative theories of gravity, J. Cosmology Astropart. Phys. 1011 (2010), 008.

[7] T. Biswas, E. Gerwick, T. Koivisto and A. Mazumdar, Towards singularity and ghost free theories of gravity, Phys. Rev. Lett. 108 (2012), 031-101.

[8] T. Biswas, A. S. Koshelev, A. Mazumdar and S. Yu. Vernov, Stable bounce and inflation in non-local higher derivative cosmology, J. Cosmology Astropart. Phys. 08 (2012), 024.

[9] A. S. Koshelev and S. Yu. Vernov, On bouncing solutions in non-local gravity, Phys. Part. Nucl. 43 (2012), 666-668. 
[10] I. Dimitrijevic, B. Dragovich, J. Grujic and Z. Rakic, On modified gravity, Springer Proceedings in Mathematics and Statistics 36, 2013, pp. 251-259.

[11] I. Dimitrijevic, B. Dragovich, J. Grujic and Z. Rakic, New cosmological solutions in nonlocal modified gravity, Rom. Journ. Phys. 58 (2013), 550-559.

[12] T. Biswas, A. Conroy, A. S. Koshelev, and A. Mazumdar, Generalized gost-free quadratic curvature gravity, Class. Quant. Grav. 31 (2014), 015-022.

[13] I. Dimitrijevic, B. Dragovich, J. Grujic and Z. Rakic, A new model of nonlocal modified gravity, Publications de l'Institut Mathematique 94 (2013), 187-196.

[14] I. Dimitrijevic, B. Dragovich, J. Grujic and Z. Rakic, Some power-law cosmological solutions in nonlocal modified gravity, in: Lie Theory and Its Applications in Physics, Springer Proceedings in Mathematics and Statistics, 2014, pp. 241-250.

[15] I. Dimitrijevic, B. Dragovich, J. Grujic and Z. Rakic, Constant Curvature Cosmological Solutions in Nonlocal Gravity, AIP Conference Proceedings, W. Univ. Timisoara, Fac. Phys, 2014, pp. 18-23.

[16] A. L. Erickcek, T. L. Smith and M. Kamionkowski, Solar system tests do rule out $1 / R$ gravity, Phys. Rev. D 74 (2006), 121-501.

[17] S. Deser and R. P. Woodard, Nonlocal cosmology, Phys. Rev. Lett. 99 (2007), 111-301.

[18] S. Nojiri and S. D. Odintsov, Modified non-local-F $(R)$ gravity as the key for inflation and dark energy, Phys. Lett. B 659 (2008), 821-826.

[19] E. Elizalde, E. O. Pozdeeva, S. Yu. Vernov and Y. Zhang, Cosmological solutions of a nonlocal model with a perfect fluid, J. Cosmology Astropart. Phys. 1307 (2013), 034.

[20] F. Briscese, A. Marciano, L. Modesto and E. N. Saridakis, Inflation in (super-)renormalizable gravity, Phys. Rev. D 87 (2013), 083-507.

[21] G. Calcagni, L. Modesto and P. Nicolini, Super-accelerting bouncing cosmology in assymptotically-free non-local gravity, Eur. Phys. J. C74 (2014), 29-99.

${ }^{1}$ Teacher Education Faculty,

UNIVERSITY OF BELGRADE,

Kraljice Natalije 43, Belgrade, Serbia

E-mail address: jelenagg@gmail.com 